Review, US Spelling

hharematology

Effectiveness and safety of erythropoiesis--stimulating agent use in the perioperative period

Effectiveness and safety of erythropoiesis stimulating agentESA use in the perioperative period

D. H. D. Tran et al.

Diane H-D Tran, Gordon T-C Wongt, Yee Eot Chee $\&$ Michael G Irwin

University of Hong Kong, Queen Mary Hospital, Department of Anaesthesiology, 102 Pokfulam Road, HK, Hong Kong gordon@hku.hk

University of Hong Kong, Department of Anaesthesiology, HK, Hong Kong

Introduction:

Erythropoiesis--stimulating agents (ESAs) are widely used to-in treating anzemia associated with renal failure. They are also now used perioperatively to reduce the use of allogeneic blood transfusions (ABT ) in patients undergoing surgery with anticipated high blood loss. Although they can reduce the risks associated with ABT and improve quality of life, the use of ESAs is still associated with adverse effects.

\title{
Areas covered:
}

A narrative review is provided on ESAs and a systematic review was -has been conducted to examine the current evidence for the efficacy and safety of perioperative ESAs use. A search of PubMed and Medline databases was has been performed using a combination of search terms including erythropoietin, perioperative, surgical, safety and efficacy.

\section{Expert opinion:}

Current evidence supports the use of perioperative ESAs to reduce the need for ABT. However, large studies assessing safety in anaemic patients with chronic renal disease have found adverse effects including cardiovascular, stroke and thromboembolic events. The dosing strategies used[CE1] have been varied and short term in comparison, to adequately assess whether these adverse effects can be conferred onto the perioperative population. Future research needs to address the questions of optimal dosing strategies in order to maximisze the positive effects but alseand minimisze adverse events.

Keywords: blood transfusion, erythropoiesis, erythropoietin, perioperative, surgery

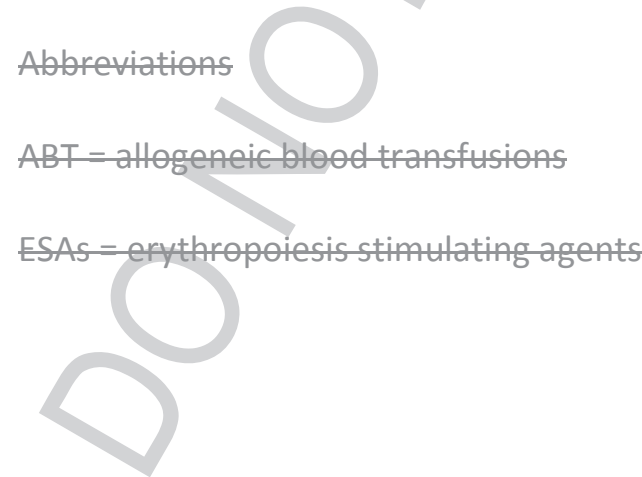


Hb- Haemoglobin

Het-Haemäotocrit

W-International Units

iv-intravenous

rHuEPO - recombinant human erythropoietin

sc-subcutaneous

\section{Introduction}

Major complex surgical procedures may be associated with substantial intraoperative and/or post operative blood loss. A significant number of patients that develop anzemia from blood loss will require allogeneic blood transfusions (ABTs) and this is more likely if preoperative anzemia is present_-, a state that is not uncommon in certain surgical populations. Anaemia in itself can be harmful; in order to maintain systemic oxygen delivery ${ }_{2}$ there is a compensatory increase in cardiac output. Even mild preoperative anaemia is independently associated with an increased risk of 30day morbidity and mortality and postoperative complication rates in patients undergoing major noncardiac surgery $[$.

Although blood transfusion, in the context of either severe anaemia or life-threatening haemorrhage $e_{2}$ can improve oxygen delivery to various organs, its use is associated with a number of well-recogniszed risks and complications. These include infectious risks, as well as transfusion reactions, transfusion errors, immunological reactions and immune modulation 2, 3. The rise in hemoglobin ( $\mathrm{Hb}$ ) from blood transfusions are-is not consistently associated with improvement in oxygen delivery or oxygen consumption. This may be due to the significantly altered properties of the stored blood, including the depletion of 2,3-DPG which causes a left shift of the oxygen dissociation curve, thus impairing the oxygen--deliveringy ability of red blood cells 4 . 
The concerns regarding the adverse effects of ABT have prompted reviews of transfusion practices and the development of strategies to minimizse the need. These include the implementation of restrictive transfusion protocols, use of pharmacological and non-pharmacological measures to reduce blood loss, preoperative autologous blood donation for transfusion, perioperative cell salvage- and stimulation of preoperative erythropoiesis 5,6 .

This review will focus on erythropoiesis_-stimulating agents (ESAs) as a component of this patient haematological strategy[CE2]. First ${ }_{2}$ it will provide-a narrative review on erythropoietin, including its history, formulations and regimens, is provided. Secondly, it will examine, in a semi-quantitative manner, the efficacy and safety of perioperative erythropoietin use is examined in a semiquantitative manner.

\subsection{AEetiology of preoperative anzemia}

In a US national audit of patients undergoing elective orthopaedic surgery, 35\% of patients were found to have $\mathrm{Hb}$ levels $<13 \mathrm{~g} / \mathrm{dL}$ at preadmission testing. Many are-were [CE3]women and anemia in approximately one--third of these patients ahad beenre the result of iron deficiency, with the remaining being attributed to chronic inflammatory disease, chronic renal disease or unknown causes 7 .

Patients undergoing major surgery with anticipated high blood loss often have co-morbidities, underlying chronic disease processes as well as being of advanced age. Aging is increasingly being identified as a proinflammatory state. The anaemia of chronic disease is multifactorial but is thought to be immune--driven. Cytokines induce changes[CE4] in iron homeostasis, impaired the proliferation of erythroid progenitor cells;_and reduce circulating levels of erythropoietin and the life-span of red blood cells. Increase uptake of iron by cells of the reticuloendothelial systems causes a diversion of iron away from the circulation. Erythropoiesis can be directly affected by the 
infiltration into bone marrow of microorganisms as well as tumour cells 8 . Ongoing occult blood losses from gastrointestinal or urogenital cancers may also contribute.

\section{Erythropoietin and erythropoiesis-stimulating agents}

Erythropoietin is the primary regulator of erythropoiesis. It is a glycoprotein hormone naturally produced and secreted primarily by renal tubular cells with a minor hepatic contribution. Production is stimulated by tissue hypoxia or severe haemorrhagic stress ${ }_{2}$ and erythropoietin binds to specific receptors on erythroid progenitor cells in the bone marrow. The ultimate effect is to increase erythropoiesis in an attempt to maintain oxygen delivery to vital organs.

In 1977, human erythropoietin was successfully purified and characteriszed from the urine of patients with aplastic anaemia. In 1985, two groups of investigators independently cloned the human erythropoietin gene, identifying the corresponding nucleotide sequences 9. Erythropoietin for clinical use is now produced by recombinant DNA technology. The first human trials using recombinant human erythropoietin (rHuEPO), which is identical to the naturally occurring erythropoietin, examined its effectiveness in correcting anzemia of chronic renal disease. These initial results demonstrated that rHuEPO could increase the haemoglobin $\underline{\mathrm{Hb}}$ level, thus removing the need for regular blood transfusion and improving the quality of life in patients requiring dialysis 10. The trial results were so impressive that rHuEPO was approved for human use in patients with chronic renal failure by the Food and Drug Administration (FDA) in June 1989 9, 11.

Erythropoietin-stimulating agents (ESAs) are given by injection to stimulate red cell production and to treat anzemia. Clinical trials have demonstrated a dose-_response relationship between erythropoietin and red blood cell expansion 12. They are commercially available in several forms. The first-generation ESAs such as Eepoetin- $\underline{\alpha}$-alfa and epoetin- $\underline{\beta}$ - beta are recombinant erythropoietin analogues, each consisting of 165 amino acids but differ only in their glycosylation. Darbepoetin- $\underline{\alpha}$-alfa, a second--generation ESA, is a hyperglycosylated derivative of Eepoetin. It has 
a longer half-life and, therefore, may be administered less frequently than Eepoetin. Although these rHuEPOs act on the same erythropoietin receptor, there are some variations on the degree of glycosylation which is responsible for the differences in pharmacokinetics and pharmacodynamics between them. The newer third_-generation ESAs are chemically synthesiszed, continuous erythrocyte receptor activators(CERA), with an even longer half--life than darbepoietin 13, 14.

The FDA has now extended approval of these agents for the treatment of anaemia resulting from a number of causes. These include chronic kidney failure, chemotherapy and certain treatments for Hhuman fimmunodeficiency $\forall \underline{v i r u s-(H I V) . ~ I t ~ i s ~ a l s o ~ u s e d ~ t o ~ r e d u c e ~ t h e ~ n u m b e r ~ o f ~ b l o o d ~}$ transfusions during and after major surgery and in patients who refuse to have an allogeneic blood transfusionABT for religious reasons such as Jehovah_'s Witness. The rRHuEPO used for these indications has been described as a 'promising blood-saving technique' 15. Preoperative rHuEPO gained regulatory approval in 1996 to reduce the need for ABT in anaemic patients (pre-treatment $\mathrm{Hb}$ of $10 \mathrm{~g} / \mathrm{dL}$ to $13 \mathrm{~g} / \mathrm{dL}$ ) undergoing major surgery 16 . It has been (along with iron, vitamin B12 and folic acid) recommended (along with iron, vitamin B12 and folic acid) as a specific medication 'tthat should be used instead of blood transfusion”- if the clinical condition of the patient permits sufficient time to promote erythropoiesis 11. The rRhuEPO has also been approved for use in patients undergoing autologous donation in Japan, Europe and Canada since 1993, 1994 and 1996, respectively, and for perioperative adjuvant therapy without autologous donation in Canada and the United States since 199617 .

Other situations relevant to the perioperative period where ESAs have been used; is to treat the anaemia associated with critically ill patients in the intensive care unit. Despite earlier trials suggesting a small decrease in ABT after administration of ESAs, a more recent multicenter trial, conducted after the more widespread use of restrictive transfusion triggers, did not show a significant reduction in ABT use 18. Trials have also been conducted in cardiac patients with acute ST elevation myocardial infarction with the aim of reducing infarct size and improving 
cardiovascular outcomes. Although these have produced positive outcomes in animal studies, so far there have not been any conclusive studies to confirm these effects in the human population 19.

The therapeutic effect of ESAs involves counteracting the antiproliferative effects of cytokines along with the stimulation of iron uptake and heme biosynthesis in erythroid progenitor cells- (see Section 1.1).

There are many approved as well as off-label uses of ESAs. The nature of the [CE5]anaemia they are treating and these range from chronic use such as to treat the anaemia of chronic renal failure as well as episodic use which would include its use to treat the anaemia of critical illness and

\section{Regimes of perioperative rHuEPO use}

Preoperative administration of rHuEPO is effective in treating anzemia by increasing the erythrocyte mass and autologous donation volumes while maintaining a higher haematocrit (Hct) 20. Consequently, it can be administered to assist with autologous donation or prior to elective surgery in patients who do not predonate. The beneficial effect of rHuEPO among patients participating in preoperative autologous blood donation programmes and for the preoperative preparation of patients has been previously reported. Early clinical trials of rHuEPO therapy in the setting of autologous donation provided further important information regarding clinical safety, rHuEPO dose, and erythropoietin response. Later trials of perioperative rHuEPO therapy without autologous donation then provided data on efficacy (reduced allogeneic blood exposure) that led to approval of rHuEPO in patients undergoing surgery. Factors that influence the response to rHuEPO include the dose and timing of treatment, combined administration of iron and baseline Hbhaemoglobin concentration $21,22$.

\subsection{Dose and timing of treatment}


The effect of rHuEPO is rapid. Within 2 to 3 days, a sustained rise in the reticulocyte index is seen and the Hcthrematocrit begins to increase. The equivalent of one unit of blood is produced by day 7 , and the equivalent of 5 units is produced by day 28 . Although several different preoperative regimens have been described, the regimen approved by the US FDA consists of four subcutaneous (s.c.) injections of epoetin- $\underline{\alpha}-$ alfa, $600 \mathrm{U} / \mathrm{kg}$ of body weight, administered at 3, 2 and 1 one week before surgery and again on the day of surgery 23. Weekly doses of rHuEPO are as effective as daily administration but are less expensive. Initiating therapy with a single weekly dose would seem logical, especially if therapy is commenced well before surgery 20.

The minimal effective rHuEPO dose required to reduce ABT rate in surgical patients is unknown, especially when administered together with iron $_{2}$ and this is reflected in the huge range of dose regimes employed by different studies. Protocols used vary from a single large dose of rHuEPO given 1 ene day preoperatively 24 and a subsequent smaller dose administered at the time of surgery 25, to multiple doses given at weekly intervals over a period of 3 to 4 weeks preoperatively 26, 27, 28. Some protocols extend into the post-operative period, whilest daily regimes administered from-up to 10 days preoperatively have also been used $29,30,31,32,33,34,35,36$. The individual single dose was higher in patients undergoing weekly treatment compared to those undergoing daily treatments, with the majority of those on weekly treatment having 40,000 IU/-per-week and those on daily treatments receiving between 10,000 世-and 21,000 IU/-daily. Some were based on weight, while others were a set dose irrespective of any other factors. Total dose is generally higher and the duration of treatment shorter in patients treated daily compared with those treated weekly.

\subsection{Route of administration}

Both the intravenous (i.v. $)$ and subeutaneous (s.c.) routes can be used to deliver rHuEPO to patients with renal impairment. The circulating half-life of rHuEPO is 6 to 8 heurs, with significant interindividual variations in plasma levels 20. Clinical studies have demonstrated that the s.c. route 


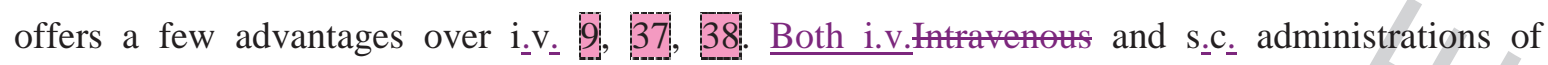
rHuEPO show substantial differences in their pharmacokinetics. Levels remain elevated longer after S.c. (48 hours) than after i.v. administration (18 - 24 hours), although peak levels are not as high. The sustained serum levels associated with S.c. dosing are more physiological and; therefore- more effectively stimulate erythropoiesis 9,20 . This pharmacokinetic difference may explain why the majority of studies chose a rHuEPO dosing strategy using the s.c. method. It is difficult then to

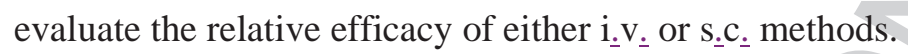

\subsection{Co-administration of iron}

Iron deficiency is considered to be the most important cause of an inadequate response to ESAs.

Erythropoietin--stimulated erythropoiesis is independent of age and gender, and the variability in response among patients is most likely attributable to iron-zrestricted erythropoiesis 17. Absolute iron deficiency, where total body iron stores are depleted, or functional iron deficiency can occur. The latter, with normal ferritin levels but low transferrin saturation, is a state which occurs when increased erythron iron requirements exceed the available supply of iron. This inability to mobilize iron stores rapidly enough develops under conditions of intense erythropoiesis such as during treatment with ESAs, and in these cases, supplementary iron may improve the response to ESAs 39. Iron deficiency may blunt the response to EPO[CE6] or delay recovery from postoperative anzemia 40. The maximal effect of rHuEPO injections is usually only achieved when patients_-' iron stores are adequate 41 .

It has been demonstrated that the use of $\mathrm{I}_{.} \mathrm{v}_{\mathbf{.}}$ iron, with or without rHuEPO, reduced the need for allogeneic blood transfusionABTs, but stimulation of erythropoiesis seemed to be more pronounced among patients also receiving rHuEPO. For these reasons, it has been recommended that rHuEPO therapy is supported by supplementary iron either orally or intravenously. Oral iron is usually effective but $i_{.} v_{-}$supplementation should be considered for patients with low iron stores, those with 
a poor initial response to rHuEPO therapy or those who demonstrate increasing evidence of iron deficiency with treatment 20. Infusion of iron should take place two to three times weekly for 3 to 4 weeks. This treatment can be administered to all patients to prevent iron-deficiency during erythropoiesis 42. Whilest the majority of studies combined treatment with iron, patients were predominantly treated with oral iron rather than i.v.

However, the use of iron supplementation in anaemia is associated with potentially deleterious effects and is therefore controversial. Iron is an essential nutrient for proliferating organisms and has been linked to increased risk of developing bacteraemia. It is also associated with the formation of oxidative free radicals which can cause tissue damage and may also have immmene modulation immunomodulatory effects[CE7] 43.

\section{Evidence for of the efficacy of ESA use}

A search was performed using PubUBMedED and OvidVID MEDLINE to identify all articles with erythropoietin as a text word. All the titles and abstracts found were examined for studies evaluating the use of, as well as safety and efficacy of ESAerythropoiesis stimulating agents in the perioperative setting. Studies were included if they were published in English between January 1993 and June 2013. Only randomiszed trials were included in this part of the review. Studies involving children and preoperative autologous blood donation were excluded. Of the 14 published studies included (see Table 1), the largest number of trials was in orthopaedic surgery, particularly joint replacement procedures, with the remaining trials in gastrointestinal cancer surgery 29, 30,31,32,33 and cardiac surgery. All were randomiszed and included eight double-blind, two single-blind and four open-label studies. The majority of primary outcomes in these trials were the need for intraoperative blood transfusion, mean number of units of blood transfused,

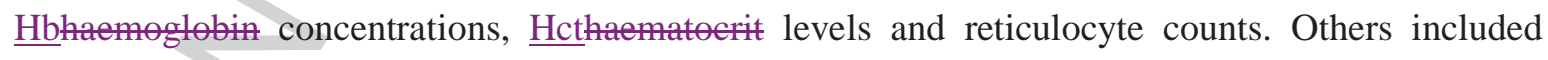
length of hospital stay and deep venous thrombosis (DVT) detected. 
On the whole, there were variations in dosage regimes and length of treatment duration between the different types of surgery. The studies looking at orthopzedic surgery generally had a weekly or daily treatment protocol that involved longer treatment duration and enrolled a larger number of patients than either cardiac or colorectal cancer surgery. In all the studies, either a minimal

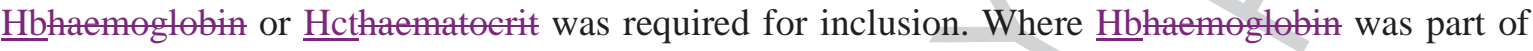
the inclusion criteria, this ranged from above $\geq 8.5 \mathrm{~g} / \mathrm{dL}$ to $10 \mathrm{~g} / \mathrm{dL}$ to below-mainly $\leq 13.5 \mathrm{~g} / \mathrm{dL}$. Two studies did set higher Hbhaemoglobin levels at $14.5 \mathrm{~g} / \mathrm{dL} 43$ and $16 \mathrm{~g} / \mathrm{dL} 34$ and for those that used Hcthaematocrit, this was set at above 42 28, 36. Most benefit was found in patients with a baseline Hbhaemoglobin of between 10 and $13 \mathrm{~g} / \mathrm{dL}$. Where stated, all studies excluded patients with uncontrolled hypertension and history of thromboembolism.

There were few standard transfusion triggers and often relied on clinical judgement of both surgeons and anzesthetists and subjective symptoms reported by patients, whilest several adopted a

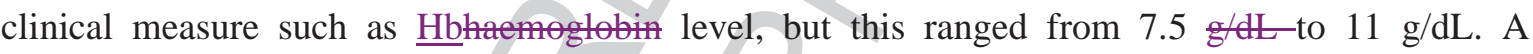
restrictive transfusion trigger has been shown to be-unlikely to be associated with an increased incidence of silent myocardial ischzemia or longer hospital stay, but may result in a significant reduction in $\mathrm{ABT}$ rate.

Three of the included studies undertaken in patients undergoing orthopaedic procedures and one in colorectal cancer surgery randomizsed patients into 3-three groups; Z-two treatment groups along with a control group 33. The 2 two treatment groups differed in dosage of rHuEPO used $;$; a higher and lower dose was studied.

Preoperative administration of rHuEPO was shown to reduce allogeneic blood exposure in individuals undergoing elective surgical procedures associated with significant blood loss such as joint replacement, cardiac and oncological surgery in all except three studies 29,30,31. All the studies showing a negative correlation with ABT rate were in patients undergoing colorectal surgery. In all those studies which showed a significant reduction of ABT associated with rHuEPO 
use, there was also a significant reduction in the mean number of units transfused in the treatment groups. In all papers that evaluated this as the primary outcome, there was also a significant increase in reticulocyte count and $\underline{\text { Hcthaematocrit. }}$

In-Among the 4 -four studies that had 2 two treatment groups as well as a control group, 3 -three $\theta$ ff thesestudies showed a significant reduction in ABT in both treatment groups. In one of these studies, there was more reduction in ABT in the lower--dose group compared to the higher dose 28 . The remaining one study-of the 4 , despite showing a reduction of ABT use in both treatment groups, was only significant with the higher dose of rHuEPO 33 .

\subsection{Colorectal cancer surgery}

There were 5-five randomiszed trials looking at the use of rHuEPO in colorectal cancer surgery. Of these, three studies 29,30,31 did not show a significant difference in blood transfusion rates between treatment group and control. One of these three studies $\operatorname{did}_{2}$ however, show a significant reduction in the mean number of units transfused in the treatment group 31. In comparison, the two other studies 32, 33 showed a significant reduction in transfusion rates in the rHuEPO group as

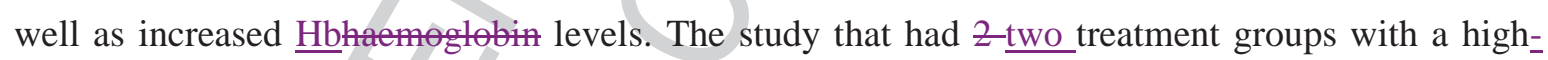
dose and low-_dose regime only showed a significant reduction in ABT in those treated with a higher dose of EPO 33 .

The rHuEPORecombinant human erythropoietin needs to be given in combination with iron $_{2}$ and this is particularly important in patients undergoing cancer surgery who may be iron--deficient, although it has been suggested that the benefit of supplemental iron may be less in oncology patients due to the decreased ability for of erythropoiesis which could be related to other factors associated with malignancy. All studies involved the co-administration of iron and in all but one, 43, this was done as oral supplementation. The studies that used i.v. iron showed a significant reduction in $\mathrm{ABT}$ in the treatment group. It appears that oncology patients respond better to i.v. 
iron supplementation in chemotherapy-induced anaemia treated with rHuEPO. This is thought[CE8] to be due to an absolute iron deficiency due tobecause of continuousing external losses externally but and also due to decreased gastrointestinal absorption and iron sequestration caused by increased expression of hepcidin that can occur in oncology patients, as well as poor compliance 8, 44. Due Owing to the shorter duration of treatment prior to surgery necessitated by the urgency of these procedures, the duration of iron treatment was has also been short.

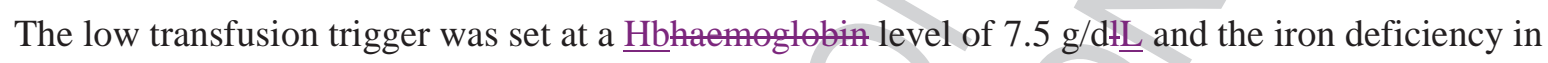
almost all patients in one of the studies may have contributed to the generally low transfusion frequency leading to a negative result 30 . Another possibility for the negative results in this group was that rHuEPO was not adequately effective in stimulating haematopoiesis in patients with tumour-induced anzemia and colorectal cancer 29. The nature of cancer surgery means that any unnecessary delay would be unethical which normally means that there is a shorter preoperative time and, therefore, a shorter period of time to initiate rHuEPO treatment. In light of this, the treatment start date in these studies ranged from 4 to 10 days prior to surgery. A daily treatment regime was used in all study protocols. This, in part, may also have contributed to the heterogeneity of the results. The differences[CE9] blood transfusion with ESA treatment is that the overall dosage of ESA administered over the treatment period was higher_; one study had the dosage of ESA for 10 days daily and the other for 7 days daily preoperatively $24,36,43$.

\subsection{Cardiac surgery}

Despite all study protocols being different with regards to dosage, interval of dosing and length of time of treatment duration, they all showed a significant reduction in ABT in the treatment group as well as significant reduction in mean units of blood transfused.

\subsection{Orthopaedic surgery}


All these studies used relatively large sample sizes, ranging from 194 patients in one study to 695 patients in another 26. All showed a significant reduction in ABT in the ESA treatment group with several also showing a significant reduction in mean units of blood transfused in the ESA group. Three studies utiliszed Ztwo treatment groups with a lower and higher dose of ESA and all showed a significant reduction in ABT in both ESA treatment groups. Dosage regimes varied from single dose to daily and weekly regimes. Total duration of treatment ranged from one single dose to 4 weeks.

\section{Safety of erythropoietin in perioperative use}

The majority of studies involving the use of perioperative erythropoietin in patients undergoing surgery were targeted at efficacy which was reflected in the primary end points being reduction in ABT or change in other hæematological parameters. Perioperative administration of ESAs to surgical patients is thought to have few adverse side effects, because it is a short-term treatment and contra-indicated for patients with co-morbidities that may predispose to these side-effects. These include uncontrolled arterial hypertension, previous acute myocardial infarction or stroke, unstable angina and severe carotid stenosis and are usually cited as exclusion criteria in these studies. Doses used in perioperative treatment tend to be lower than that which have been used conventionally in the past and also for a shorter duration of time. Dee-Owing to the nature of expected surgical blood loss, the rise in $\mathrm{Hb}$ is not sustained.

Trials did report adverse effects in their sample populations including deep venous thrombosis (DVT), hypertension, infection, anastomotic breakdown and death. However, due to the low incidence of such events in the studies, no clear correlation with rHuEPO treatment could be made. The rRhuEPO was also withheld in patients who developed any of these adverse events during the study period. A concern for EPO use is the development of thrombotic complications associated

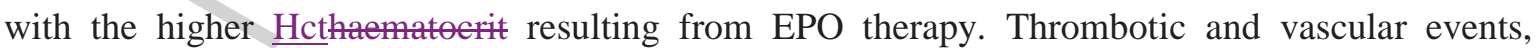
including myocardial infarction, angina, deep vein thrombosis, superficial phlebitis; and peripheral 
arterial thrombosis are associated with rapid increases in the Hbhaemoglobin level and the Hcthaematocrit and are of special concern in patients who are managed with EPO. None of the studies clearly stated whether anticoagulation prophylaxis was routinely used in their sample population.

The safety effects of rHuEPO have been more extensively studied in other non-surgical patient populations. Three large randomiszed controlled trials, the Normal Haematocrit Study 45, the Correction of Hemoglobin and Outcomes in Renal Insufficiency (CHOIR) Trial 46 and The Trial to Reduce Cardiovascular Events with Aranesp Therapy (TREAT) 47 46 involved patients with anzemia and chronic kidney disease. These studies showed that the use of ESAs to achieve a higher Hbhaemoglobin level rather than partial correction of anaemia, which is the most common use of ESAs in this population, was associated with significantly increased adverse events including non fatal and fatal myocardial infarction, non-fatal and fatal stroke, death and thromboembolic events. In fact, the results were so conclusive that $z$ two of the studies were halted early. In these studies, the dose of ESA was adjusted and increased if the target Hb was not achieved during monitoring. A review of these studies suggested that the increased incidence of adverse events may be related to the rapidity of increase in Hbhaemoglobin concentration and an overshoot of target concentration which may have been due to aggressive dosing. Another possibility was that these adverse effects may be due to some other consequence of ESAs such as trophic effects on vascular endothelial or smooth muscle cells 48 .

Studies of the use of ESAs in critically ill patients showed that the proportion of patients who experienced thrombotic events was significantly greater with rHuEPO than placebo. However, the risk for of thrombotic events was significantly increased in patients who did not receive heparin at baseline but not among patients who did receive heparin at baseline 18 .

In a large multicentere trial of 680 surgical patients scheduled for elective spine surgery, blood transfusion and patient outcomes were compared for ESA and placebo-treated cohorts. These 
patients did not receive anticoagulation prophylaxis for thrombotic adverse events. This study documented a higher incidence of deep vein thrombosis of $4.7 \%$ in the study group was $4.7 \%$ compared to $2.1 \%$ in control-2.1\%, with the upper confidence limit for the between-group difference being $5.4 \%$. This exceeded the predefined boundary of $4 \%$ that was required to demonstrate noninferiority 49 .

In a small-_scale ${ }_{2}$ double-blind, placebo--controlled study involving 30 healthy male volunteers who were given rHuEPO, there was a moderate stimulation of thrombopoiesis and this has been suggested to be increased by $15 \%$. However, rHuEPO was also thought to cause increased platelet reactivity and a thrombogenic effect on the newly synthesized platelets, which may lead to increased thrombotic events 12 .

Hypertension is commonly associated with long-term rHuEPO therapy in patients with chronic renal failure. Although there seems to be a low risk of precipitating hypertension during shortcourse preoperative rHuEPO therapy, there have been reports of individual patients without prior history developing hypertension during such treatment. Despite the finding of hypertension in some of the patients in these studies, there was no clear correlation with rHuEPO therapy.

There is uncertainty about the potential side effects of erythropoietin analogues in anemic patientseople with anaemia-who are receiving treatment for cancer. The European Medicines Agency 'has recently reviewed the safety of erythropoietin analogues based on new data from both published and unpublished studies. These studies suggest an increased risk of serious cardiovascular complications in people with chronic renal failure and a possible effect on tumour progression in people with cancer' 13. However, the risk/benefit of ESAs has recently been questioned based epon individual reports and meta-analyses showing that these agents are associated with an increased risk of mortality when chronically administered to patients with advanced/metastatic cancers 50. Erythropoietin receptors are found on several malignant lines and can increase tumour recurrence rates. 
Although the positive short-term effects of ESA therapy with ESAs on the correction of anaemia and avoidance of blood transfusions are well documented, few data are available on possible effects on the course of underlying disease, particularly since ESAs can exert additional biological effects including interference with the signal transduction cascade of cytokines.

\section{Conclusions}

Minimiszing allogeneic blood transfusion $\underline{A B T}$ should be a high priority of any health-care delivery system. Transfusions associated with perioperative care represent a significant proportion of blood consumed. The concept of patient blood management has recently been described and was adopted by the World Health Organiszation in 2010 as a principle to improve transfusion safety. It refers to pre-empting and significantly reducing the need for transfusion by addressing anaemia, blood loss and hypoxia as modifiable risk factors. It comprises three main factors: detection and correction of preoperative anaemia, minimiszing perioperative blood loss and optimiszing the patient'-s physiological tolerance to anzemia 50. It can play a significant role in negating preoperative anzemia and augmenting the quantity of blood available for autologous transfusion.

The effect of ESAs on transfusion requirements in cancer surgery patients remains uncertain, although, in patients who underwent orthopaedic surgery, treatment with preoperative ESA reduced both the use and rate of blood transfusion. It is clear that the use of perioperative rHuEPO does reduce the number of transfusions, reduce the mean number of transfusions given, increase the

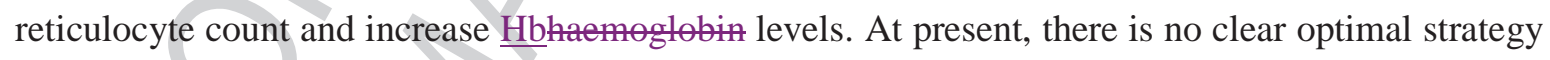
with respect to dose, timing and length of treatment. The subeutaneous-s.c. route is commonly used and has pharmacokinetic advantages. Perioperative rHuEPO therapy seems to be associated with a low incidence of complications, although larger studies are necessary to define this more clearly. There is an apparent risk of deep vein thrombosis but the risk can be ameliorated with the judicious use of anticoagulation. 
Despite being theoretically attractive, the uptake of perioperative rHuEPO use has been slow. One reason for the limited use of preoperative rHuEPO in clinical practice may be the impracticality of the dosing schedule as recommended by efficacy trials, particularly with a required lead-_time of 4 weeks. Another reason may be the high-cost of the recommended dosage. Complications from

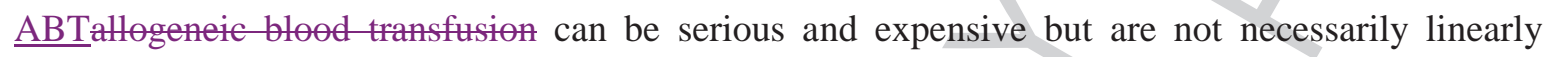
related to the numbers of units transfused and, consequently, the cost savings gained from averting a disaster are difficult to quantify. Other effects such as immune suppression are even less tangible. The 'true' cost_-effectiveness of EPO treatment is thus difficult to calculate and may be a hindrance to more widespread adoption.

\section{Expert opinion}

There is evidence that the perioperative use of ESAs can reduce the need for ABTallogeneic blood transfusions in patients undergoing major surgery with high anticipated high blood loss. With the current drive to improve transfusion safety by modifying risk factors such as anzemia, blood loss and hypoxia, the targeting of anzemic patients preoperatively and the use of ESAs to stimulate erythropoiesis have been shown to have beneficial effects. The increase in haematological parameters may improve a patient_'s status in order to optimisze post-operative rehabilitation, which can in turn lead to reduced hospital stay and improved quality of life.

Currently, the use of perioperative ESAs in surgery with anticipated high blood loss does not appear to be widely adopted. Whilest the evidence exists for the use of rHuEPO in the perioperative setting, it has yet to be quantified whether other types of ESAs may also be effective. Development in-of the newer ESAs with their more favourable pharmacokinetic profiles may mean decreased frequency of administration and may therefore improve the practicalities of incorporating ESAs into a perioperative programme.

Many of the studies were in small numbers of patients and it is difficult to adequately assess efficacy as an end_point. Safety of ESAs have mainly come from the large--scale studies in anzemic 
patients with chronic renal disease which have shown an increased risk of non-fatal and fatal myocardial infarction, non-fatal and fatal stroke, death and thromboembolic events. It is unclear though whether these results can be extrapolated to the perioperative population. There are many

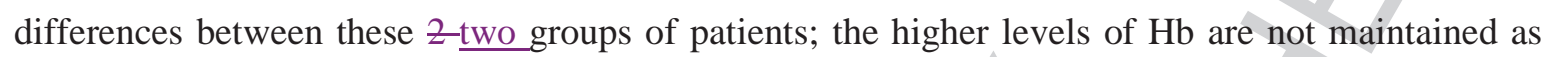
significant blood loss is expected intraoperatively in the perioperative group and the duration is $\underline{\text { of }}$ treatment is much shorter with much lower cumulative doses. The elective surgical population will also have a better functional and physiological baseline.

Well_-designed prospective randomized controlled trialRCFs on a larger scale are needed to address dose, type of ESA, timing and duration of administration in order for optimal dosing strategies to be formulated. The adverse event reporting could also be more accurate.

Once an optimal dosing strategy can be agreed, it would be useful to undertake cost_-effectiveness studies; there are not only the costs associated with the treatment drug itself, but also there are administration and screening costs, but these may be ameliorated by the cost savings of reduction in hospital stay as well as improvement in quality of life.

An important area for future research may be to explore further the pleiotropic effects of ESAs as well as to investigate the effects of ESAs at the cellular level as possible mechanisms to exert their adverse effects.

Given the awareness of risks and complications associated with ABTallogeneic blood transfusions, the use of perioperative ESA in surgery with anticipated high blood loss; is an important consideration.

Article highlights.

Benefits of perioperative erythropoiesis stimulating agents (ESAs) include an increase in Hcthaematocrit and reticulocyte counts which lead to a reduction in ABTallogeneic blood transfusions.

Reduction in ABTallogeneic blood transfusion haše been consistently shown in randomized trials of patients undergoing orthopaedic and cardiac surgery.

Use of perioperative ESAs in cancer patients hašte not consistently demonstrated a reduction in ABTallogeneic blood transfusion use. 
Iron deficiency can reduce the efficacy of ESAs.

The optimal dosing strategies for perioperative use have not yet been defined.

ESA use in chronic renal failure patients with anzemia have shown an increase in adverse events when aiming for normalization of Hbhaemoglobin levels rather than partial correction.

Adverse events associated with short-_term perioperative use have not yet been quantified.

This box summarizes key points contained in the article.

\section{Declaration of interest}

The authors have no competing interests to declare [r10]and have received no funding in preparation of the manuscript.

Table 1. Summary of the clinic trials included in this review.

\begin{tabular}{|c|c|c|c|c|c|c|c|c|c|}
\hline $\begin{array}{l}\text { Type of } \\
\text { surgery }\end{array}$ & \begin{tabular}{|c|} 
Type of \\
blinding
\end{tabular} & $\begin{array}{l}\text { No. of } \\
\text { patients }\end{array}$ & $\begin{array}{c}\text { Start } \\
\mathrm{Hb}\end{array}$ & $\begin{array}{l}\text { ESA } \\
\text { used }\end{array}$ & $\begin{array}{l}\text { Treatment } \\
\text { duration } \\
\text { prior to } \\
\text { surgery }\end{array}$ & $\begin{array}{c}\text { Total } \\
\text { duration } \\
\text { treatment }\end{array}$ & $\begin{array}{c}\text { Administration } \\
\text { frequency }\end{array}$ & $\begin{array}{l}\text { Fe } \\
\text { used }\end{array}$ & t \\
\hline $\begin{array}{l}\text { Orthopaedic - } \\
\text { bilateral knee } \\
\text { replacements) } \\
25\end{array}$ & өOpen & 108 & $\begin{array}{l}\mathrm{Hb} \\
>10\end{array}$ & $\begin{array}{l}\text { EPO B } \\
\text { ses.C. }\end{array}$ & $\begin{array}{l}\text { Once } \\
\text { during } \\
\text { surgery }\end{array}$ & $\begin{array}{l}\text { Single } \\
\text { dose }\end{array}$ & Single dose & $\begin{array}{l}\text { Ivi.v. } \\
\text { Fe } \\
\text { sucrose }\end{array}$ & $\begin{array}{l}1 \mathrm{uni1} \\
69 \mathrm{~g} / \\
\text { betwe }\end{array}$ \\
\hline $\begin{array}{l}\text { Orthopaedic - } \\
\text { primary or } \\
\text { revision hip } 34\end{array}$ & ADouble & 320 & $\begin{array}{l}\mathrm{Hb} \\
11- \\
16\end{array}$ & $\begin{array}{l}\text { EPO } \\
\text { S.C.SE }\end{array}$ & 10 days & 14 days & dDaily & $\begin{array}{l}\text { Oral } \\
\text { Fe } \\
\text { sulfate }\end{array}$ & \begin{tabular}{|l|} 
Clini \\
pract \\
volun \\
intrac \\
9/dL \\
opera
\end{tabular} \\
\hline $\begin{array}{l}\text { Orthopaedic - } \\
\text { all elective } \\
\text { major } 26\end{array}$ & $\theta$ Open & 695 & $\begin{array}{l}\mathrm{Hb} \\
10- \\
13\end{array}$ & \begin{tabular}{|l|} 
EPO A \\
s.c.se
\end{tabular} & 21 days & 22 days & wWeekly & $\begin{array}{l}\text { Oral } \\
\text { Fe }\end{array}$ & $\mathrm{Hb}<$ \\
\hline $\begin{array}{l}\text { Orthopaedic - } \\
\text { major elective } \\
35\end{array}$ & ADouble & 200 & & \begin{tabular}{|l} 
EPO \\
s.c.se \\
(3 \\
groups)
\end{tabular} & 10 days & 15 days & eDaily & $\begin{array}{l}\text { Oral } \\
\text { Fe }\end{array}$ & \begin{tabular}{|l} 
Discr \\
surge \\
0.27
\end{tabular} \\
\hline $\begin{array}{l}\text { Orthopaedic - } \\
\text { total hip } \\
\text { arthroplasty } 27\end{array}$ & Double & 201 & $\begin{array}{l}\mathrm{Hb} \\
9.8 \\
- \\
13.7 \\
\end{array}$ & $\begin{array}{l}\text { EPO A } \\
\text { S.c.se } \\
\text { (3 gps) }\end{array}$ & 4 weeks & 4 weeks & Wweekly & $\begin{array}{l}\text { Oral } \\
\text { Fe }\end{array}$ & $\begin{array}{l}\text { Discr } \\
\text { surge }\end{array}$ \\
\hline
\end{tabular}




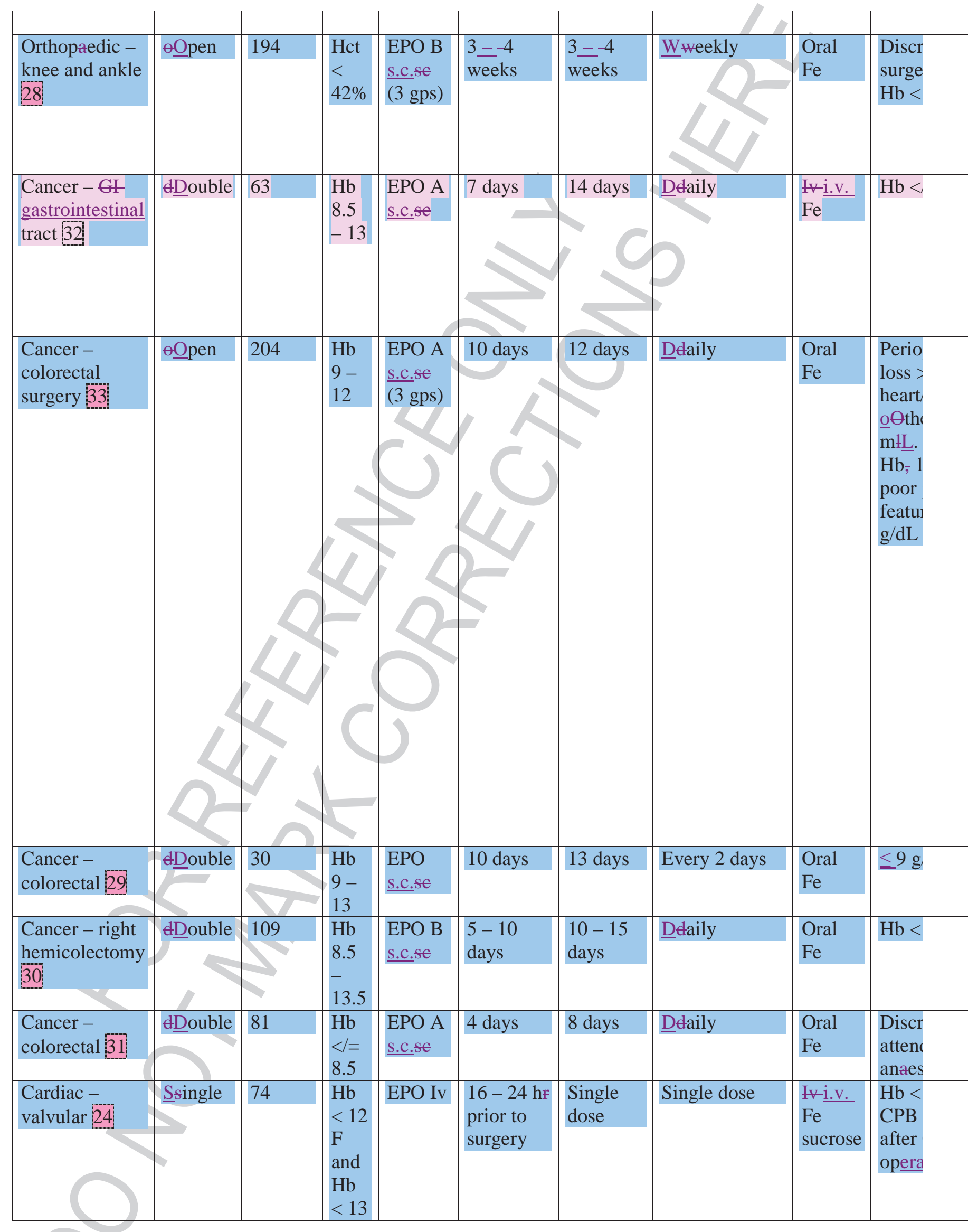




\begin{tabular}{|c|c|c|c|c|c|c|c|c|c|}
\hline & & & $\mathrm{M}$ & & & & $7 x$ & & \\
\hline $\begin{array}{l}\text { Cardiac - off } \\
\text { pump CABG } \\
43\end{array}$ & $\underline{\text { Ssingle }}$ & 320 & $\begin{array}{l}\mathrm{Hb} \\
< \\
14.5\end{array}$ & $\begin{array}{l}\text { EPO } \\
\text { s.C.SE }\end{array}$ & 2 days & 5 days & Dedaily & - & $\mathrm{Hb}<$ \\
\hline $\begin{array}{l}\text { Cardiac - open } \\
\text { heart } 36\end{array}$ & Ddouble & 76 & $\begin{array}{l}\text { Hct } \\
< \\
0.45\end{array}$ & $\begin{array}{l}\text { EPO B } \\
\text { ivi.v. }\end{array}$ & 14 days & 14 days & $5 \mathrm{do}$ & $\begin{array}{l}\text { Oral } \\
\text { Fe } \\
\text { sulfate }\end{array}$ & $\begin{array}{l}\text { CPB: } \\
\text { posto } \\
8.5 \mathrm{~g} \\
0.26\end{array}$ \\
\hline
\end{tabular}

ABT: Allogeneic blood transfusion; CABG: Coronary artery bypass graft; CPB: Cardiopulmonary bypass; EPO A: Epoetin- $\alpha$-alfa; EPO B: Epoetin- $\underline{\beta}$ beta; ESA: Erythropoiesis--stimulating agents; Fe: Iron; Hb: Haemoglobin; Hct: Haemocrit; HD: High dose, 1.v.: Intravenous, LD: Low dose; s.c.: Subcutaneous.

Bibliography

Papers of special note have been highlighted as either of interest $(\cdot)$ or of considerable interest $(\cdot \cdot)$ to readers.

$<$ journal $>1$. Meybohm P, Zacharowski K. Preoperative anaemia and postoperative outcomes in noncardiac surgery. Lancet 2011;378:1396.

$<$ journal>2. Vamvakas EC, Blajchman MA. Transfusion-related mortality: the ongoing risks of allogeneic blood transfusion and the available strategies for their prevention. Blood 2009;113:3406-17.

$<$ journal $>3$. Hendrickson JE, Hillyer CD. Noninfectious serious hazards of transfusion. Anesth Analg 2009;108:759-69.

$<$ journal $>4$. Ho J, Sibbald WJ, Chin-Yee IH. Effects of storage on efficacy of red cell transfusion: when is it not safe? Crit Care Med 2003;31(12):S687-97.

$<$ journal $>5$. Munoz M, Garcia-Erce JA, Villar I, Thomas D. Blood conservation strategies in major orthopaedic surgery: efficacy, safety and European regulations. Vox Sang 2009;96:1-13.

$<$ journal $>6$. Fullerton DA, Campbell DN, Whitman GJ. Use of human recombinant erythropoietin to correct severe preoperative anemia. Ann Thorac Surg 1991;51:825-6.

$<$ journal > 7. Goodnough LT, Maniatis A, Earnshaw P. Detection, evaluation and management of preoperative anaemia in the elective orthopaedic patient: NATA guidelines. BJA 2011;106:13-22.

$<$ journal>8. Weiss G, Goodnough LT. Anemia of Chronic Disease. NEJM 2005;352(10):1011-23.

$<$ journal>9. Ng T, Marx G, Littlewood T, Macdougall I. Recombinant erythropoietin in clinical practice. Postgrad Med J 2003;79:367-76.

$<$ journal $>10$. Goodnough LT, Shander A. Current status of pharmacologic therapies in patient blood management. Anesth Analg 2013;116:15-34.

$<$ journal $>11$. Goodnough LT, Monk TG, Andriole GL. Erythropoietin therapy. N Engl J Med 1997;336:933-8.

$<$ journal $>12$. Stohlawetz PJ, Dzirlo L, Hergovich N, et al. Effects of erythropoietin on platelet reactivity and thrombopoiesis in humans. Blood 2000 May 1;95(9):2983-9.

$<$ other $>13$. NICE Guidelines. Epoetin alfa, epoetin beta and darbepoetin alfa for cancer treatmentinduced anaemia, Reviewed 2009.

$<$ journal>14. Macdougall IC. Novel erythropoiesis-stimulating agents: a new era in anemia management. Clin J Am Soc Nephrol 2008;3(1):200-7.

$<$ journal $>15$. Qureshi M, Momoh I, Bankes M, et al. Erythropoietin provides a useful strategy for treating preoperative anemia in planned elective orthopedic surgery: an analysis of benefit in routine practice. Transfusion 2012;52:2063-4.

$<$ journal >16. Marcucci C, Madjdpour C, Spahn DR. Allogeneic blood transfusions: benefit, risks and clinical indications in countries with a low or high human development index. $\mathrm{Br}$ Med Bull 2004;70:15-28.

$<$ journal>17. Goodnough LT, Monk TG. Erythropoietin therapy in the perioperative setting. Clin Orthop 
Relat Res 1998:82-8.

$<$ journal $>18$. Corwin HL, Gettinger A, Fabian TC, et al. Efficacy and safety of epoetin alfa in critically ill patients. NEJM 2007;357:965-76.

$<$ journal>19. Najjar SS, Rao SV, Melloni C, et al. Intravenous erythropoietin in patients with STsegment elevation myocardial infarction. JAMA 2011;305:1863-1872.

$<$ journal>20. Crosby E. Perioperative use of erythropoietin. Am J Ther 2002;9:371-6.

$<$ journal>21. Kiyama H, Ohshima N, Imazeki T, Yamada T. Autologous blood donation with recombinant human erythropoietin in anemic patients. Ann Thorac Surg 1999;68:1652-6.

$<$ journal>22. Price TH, Goodnough LT, Vogler WR, et al. The effect of recombinant human erythropoietin on the efficacy of autologous blood donation in patients with low hematocrits: a multicenter, randomized, double-blind, controlled trial. Transfusion 1996;36:29-36.

$<$ journal>23. Goldberg MA, McCutchen JW, Jove M, et al. A safety and efficacy comparison study of two dosing regimens of epoetin alfa in patients undergoing major orthopedic surgery. Am J Orthop (Belle Mead NJ) 1996;25:544-52.

$<$ journal $>24$. Yoo YC, Shim JK, Kim JC, et al. Effect of single recombinant human erythropoietin injection on transfusion requirements in preoperatively anemic patients undergoing valvular heart surgery. Anesthesiology 2011;115:929-37.

$<$ journal>25. Na HS, Shin SY, Hwang JY, et al. Effects of intravenous iron combined with low-dose recombinant human erythropoietin on transfusion requirements in iron-deficient patients undergoing bilateral total knee replacement arthroplasty. Transfusion 2011;51:118-24.

$<$ journal>26. Weber EW, Slappendel R, Hemon Y, et al. Effects of epoetin alfa on blood transfusions and postoperative recovery in orthopaedic surgery: the European Epoetin Alfa Surgery Trial (EEST). Eur J Anaesthesiol 2005;22:249-57.

$<$ journal>27. Wurnig C, Schatz K, Noske H, et al. Subcutaneous Low-Dose Epoetin Beta for the Avoidance of Transfusion in Patients Schedules for Elective Surgery Not Eligible for Autologous Blood Donation. Eur Surg Res 2001;33:303-310.

$<$ journal $>28$. Feagan BG, Wong CJ, Kirkley A, et al. Erythropoietin with iron supplementation to prevent allogeneic blood transfusion in total hip joint arthroplasty. A randomized, controlled trial. Ann Intern Med 2000;133:845-54.

$<$ journal $>29$. Heiss MM, Tarabichi A, Delanoff $C$, et al. Perisurgical erythropoietin application in anemic patients with colorectal cancer: a double-blind randomized study. Surgery 1996;119:5237.

$<$ journal $>30$. Kettelhack C, Hones C, Messinger D, Schlag PM. Randomized multicentre trial of the influence of recombinant human erythropoietin on intraoperative and postoperative transfusion need in anaemic patients undergoing right hemicolectomy for carcinoma. Br J Surg 1998;85:63-7.

- This randomized, double-blind multicentere trial did not show a significant reduction in blood transfusions in patients treated with rHuEPO. A Hb trigger of less than $\leq 7.5 \mathrm{~g} / \mathrm{dL}$ was used for transfusion.

$<$ journal > 31. Qvist N, Boesby S, Wolff B, Hansen CP. Recombinant human erythropoietin and hemoglobin concentration at operation and during the postoperative period: reduced need for blood transfusions in patients undergoing colorectal surgery-prospective double-blind placebocontrolled study. World J Surg 1999;23:30-5.

$<$ journal $>32$. Kosmadakis N, Messaris E, Maris A, et al. Perioperative erythropoietin administration in patients with gastrointestinal tract cancer: prospective randomized double-blind study. Ann Surg 2003;237:417-21.

- This randomized, double-blind trial showed a significant reduction in blood transfusions in rHuEPO--treated patients and used supplementary i... i. iron.

$<$ journal>33. Christodoulakis M, Tsiftsis DD. Preoperative epoetin alfa in colorectal surgery: a randomized, controlled study. Ann Surg Oncol 2005;12:718-25.

- This large randomized, controlled study of 204 patients showed a significant reduction in blood transfusion rates in patients treated with a higher dose of rHuEPO. 
$<$ journal $>34$. Effectiveness of perioperative recombinant human erythropoietin in elective hip replacement. Canadian Orthopedic Perioperative Erythropoietin Study Group. Lancet[r12] 1993;341:1227-32.

•• This large prospective multicentere ${ }_{2}$ double--blind ${ }_{2}$ randomized trial was the first to show a reduction of ABT in perioperative patients undergoing orthopaedic surgery.

$<$ journal $>35$. Faris PM, Ritter MA, Abels RI. The effects of recombinant human erythropoiefin on perioperative transfusion requirements in patients having a major orthopaedic operation. The American Erythropoietin Study Group. J Bone Joint Surg Am 1996;78:62-72.

$<$ journal $>36$. Sowade $O$, Warnke $H$, Scigalla P, et al. Avoidance of allogeneic blood transfusions by treatment with epoetin beta (recombinant human erythropoietin) in patients undergoing openheart surgery. Blood 1997;89:411-8.

$<$ journal $>37$. Eidemak I, Friedberg MO, Ladefoged SD, et al. Intravenous versus subcutaneous administration of recombinant human erythropoietin in patients on haemodialysis and CAPD. Nephrol Dial Transplant 1992;7:526-9.

$<$ journal $>38$. Horl WH. Optimal route of administration of erythropoietin in chronic renal failure patients: intravenous versus subcutaneous. Acta Haematol 1992;87(Suppl 1):16-9.

$<$ journal $>39$. Goodnough LT. Iron deficiency syndromes and iron-restricted erythropoiesis. Transfusion 2012;52:1584-1592.

$<$ journal $>40$. Aderinto J, Brenkel IJ. Pre-operative predictors of the requirement for blood transfusion following total hip replacement. J Bone Joint Surg Br 2004;86:970-3.

$<$ journal> 41. Bacuzzi A, Dionigi G, Piffaretti G, et al. Preoperative methods to improve erythropoiesis. Transplant Proc 2011;43:324-6.

$<$ journal $>42$. Piagnerelli M, Vincent J-L. The use of erythropoiesis-stimulating agents in the intensive care. Crit Care Clin 2012;28:345-362.

$<$ journal $>43$. Weltert L, D'Alessandro S, Nardella S, et al. Preoperative very short-term, high-dose erythropoietin administration diminishes blood transfusion rate in off-pump coronary artery bypass: a randomized blind controlled study. J Thorac Cardiovasc Surg 2010;139:621-6; discussion 6-7.

$<$ journal $>44$. Wingard RL, Parker RA, Ismail N, Hakim RM. Efficacy of oral iron therapy in patients receiving recombinant human erythropoietin. Am J Kidney Dis 1995;25:433-9.

$<$ journal $>45$. Besarab A, Bolton WK, Browne JK, et al. The effects of normal as compared with low hematocrit values in patients with cardiac disease who are receiving hemodialysis and epoetin. $N$ Engl J Med 1998;339:584-90.

$<$ journal $>46$. Singh AK, Szczech L, Tang KL, et al. Correction of anemia with epoetin alfa in chronic kidney disease. N Engl J Med 2006;355:2085-98.

- Open-label-2 randomized trial enrolling 1432 patients with anzemia and chronic renal disease showed that a target $\mathrm{Hb}$ of $13.5 \mathrm{~g} / \mathrm{dL}$ compared with $11.3 \mathrm{~g} / \mathrm{dL}$ was associated with an increased risk; this trial was halted early.

$<$ journal $>47$. Pfeffer MA, Burdmann EA, Chen CY, et al. A trial of darbepoetin alfa in type 2 diabetes and chronic kidney disease. N Engl J Med 2009;361:2019-32.

- Large randomized trial involving 4038 patients with diabetes, chronic kidney disease and anemia showed that darbepoetin did not reduce the risk of death, cardiovascular event but increased the risk of stroke.

$<$ journal $>48$. Unger EF, Thompson AM, Blank MJ, Temple R. Erythropoiesis-stimulating agents-time for a reevaluation. N Engl J Med 2010;362:189-92.

- Interesting review on the current status of ESAs highlighting the major concerns regarding ESAs to increase $\mathrm{Hb}$ in patients with chronic kidney disease above a level intended solely to avert the need for blood transfusions.

$<$ journal $>49$. Stowell CP, Jones SC, Enny C, et al. An open-label, randomized, parallel-group study of perioperative epoetin alfa versus standard of care for blood conservation in major elective spinal surgery: safety analysis. Spine (Phila Pa 1976) 2009;34:2479-85. 
•• This large study involving 680 patients documented a higher incidence of deep venous thrombosisDVT in those patients who did not receive prophylactic anticoagulation before spinal surgery.

$<$ journal $>50$. Shander A, Van Aken H, Colomina MJ, et al. Patient blood management in Europe. $\mathrm{Br} \mathrm{J}$ Anaesth 2012;109:55-68. 